\title{
Artificial Intelligence Based Face Mask Detection System
}

\author{
Vivek Kumar Pandey DIAT, Pune
}

\begin{abstract}
With the advent of COVID-19 pandemic, use of mask is mandatory as per WHO/ ICMR guidelines to avert spread of CORONA virus. The post lockdown period has seen increase in cases day by day as people have now stepped out of their home to resume their work and recreational activities. Wearing mask all the time has still not found an enduring place in our day to day routine practices. It is a natural human tendency to be complacent and to remove mask while talking, working or after prolong use just use to relax and breathe properly. Thus not only risking own life but also of others who might have come in contact with the person during the period when he/she was not wearing mask. Presently the inspection of people with/ without mask is being done manually and visually by sentries/ guards present at entry/ exit points. Guards/ Sentries cannot be stationed at every place to keep a check on such people who remove their mask and roam around without restraint once they have been scrutinized at the entry gate. In the proposed system, efforts have been made in inspecting people with/ without mask automatically with the help of Computer vision and Artificial Intelligence. This module detects the face of the individual, identifies whether he/she is wearing mask or not and raises an alarm if the person is detected without wearing mask.
\end{abstract}

Keywords:- Face mask detection, Artificial Intelligence, Computer vision.

\section{INTRODUCTION}

With the ongoing post lockdown period after COVID19 , use of mask is obligatory for everybody, not only for self protection but also to prevent spread of the virus. Complacency tends to creep in people to adhere to laid down restriction/ advice for protracted time and thereafter they maneuver their own diluted version of the guidelines to best suit one's comfort levels. Hence there sneak in the tendency to remove mask as per their convenience and wear it again as per their comfort. Therefore not only risking own life but also of several people who might come in contact with this person knowingly/ unknowingly. This module uses Artificial Intelligence to automatically detect a person with/ without wearing mask and triggers an alarm when a person is detected without wearing a mask.

\section{OBJECTIVE}

The primary objective of this service paper is introduce an automated module based on Artificial Intelligence which automatically detects a person without wearing mask and triggers an alarm when such a person is detected. This will help in preventing the spread of the pandemic and will aid in avoiding complacency in people to wear mask at all times when out of their home.

\section{BRIEF DESCRIPTION}

The need of mask detection module arises because now the offices/establishment is open to all. Therefore there is currently unhindered movement of people in/out of establishment/office areas. As per ICMR guidelines, use of Face Mask must for everyone (Mandatory) for all. It is a natural human tendency to put on the mask before inspection (in/out gate guarded by sentries) and removal post inspection (just to relax and breathe easily). Now this is a clear violation of safety measures and Social Distancing norms. Well at entry/ points, body temperature can be monitored using thermal thermometer and status of Aaraoya setu app can also be verified but this does not guarantee that the person is Covid positive/ negative. As such, the person cannot move unobstructed without wearing a mask even after entering the campus. Moreover Sentries/ Guards cannot be stationed at every corner of establishment to keep a check on people who remove their mask and roam around liberally unobserved assuming that nobody can catch hold of them once they have crossed in/out gate guarded by sentries. This module will identify people entering the campus with/ without mask and entry to individuals without mask can possibly be denied. Whereas inside campus, the suitable placed device will be able to detect people not wearing their mask easily and then they can be reminded to put on their mask again. They also can be adequately warned so that they do not repeat this mistake again thus endangering lives of others as well as their own. This will also act as a much needed deterrent.

\section{A. Methodology}

The Methodology used to create this module using Artificial Intelligence is as follows:-

$>$ Training an AI based model to detect and distinguish human faces with and without masks.

$>$ Capture face from live video streaming/ images with help of AI algorithm

$>$ Extracting relevant features from detected portion of face 
Apply the trained model to identify and distinguish faces with and without mask

$>$ Raise an alarm on detection of faces without mask on.

\section{B. Workflow diagram and sequence of action}

Here at first, a model is trained with hundreds of images both of people wearing mask and not wearing mask. The model learns to distinguish faces with/ without mask.
Once trained, the model is saved and when it is presented with new set of faces via images or live video, it extracts ROI from the face and based on its learning accurately detects faces with and without mask. The algorithm then raises an alarm when a person is detected without wearing a mask. The workflow and steps involved in the process is described with the help of a flow diagram as shown below:

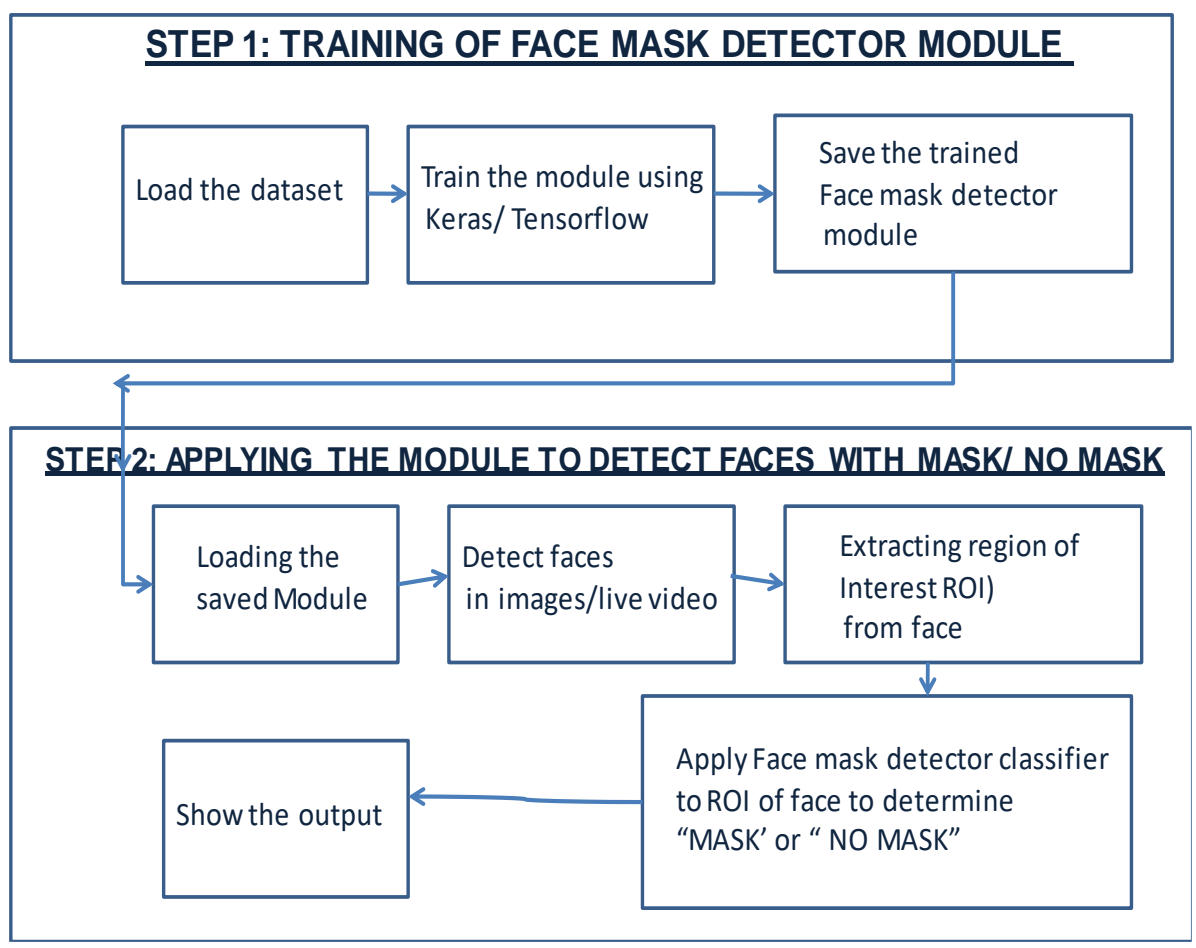

Fig 1

\section{TRIALS AND OUTPUT}

After successful compilation of the module, it was tested on various images/ live video of people wearing mask and without mask. The module was able to successfully detect faces with and without mask on. Snapshot of output of model is as below:-

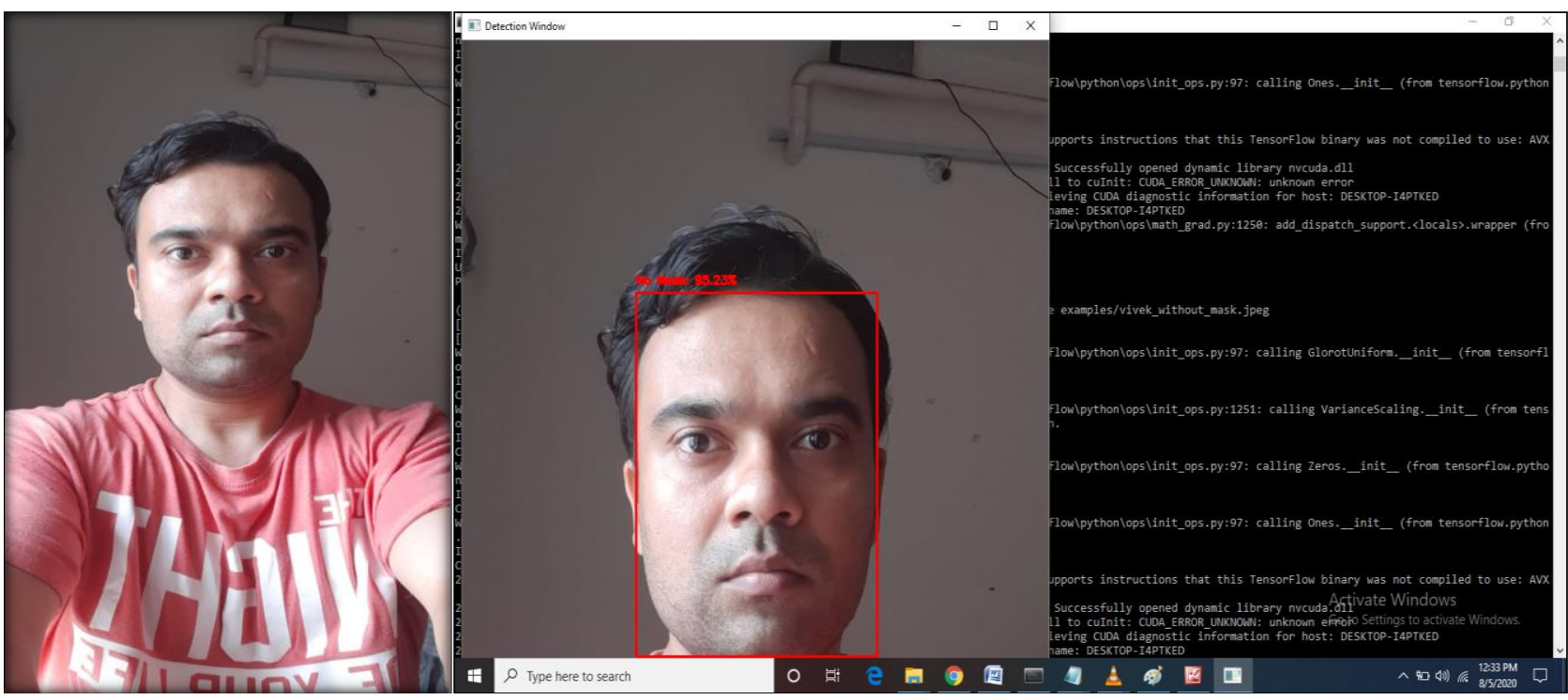




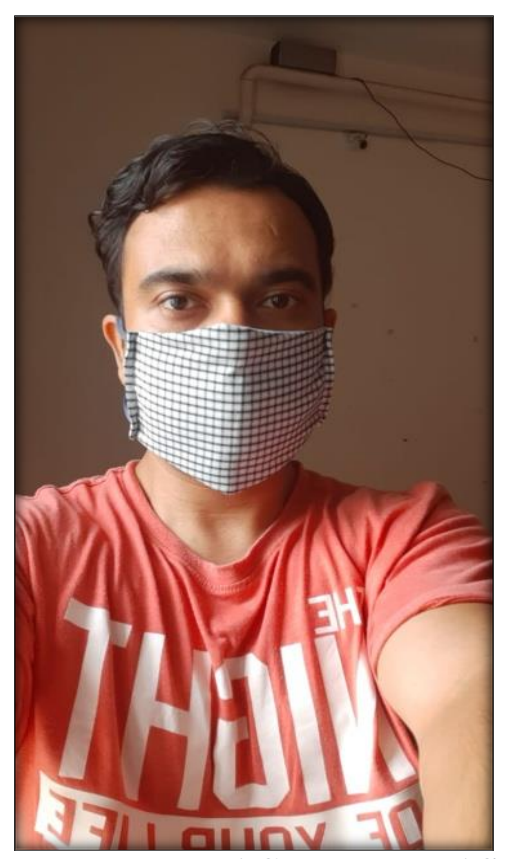

INPUT IMAGE WITH MASK

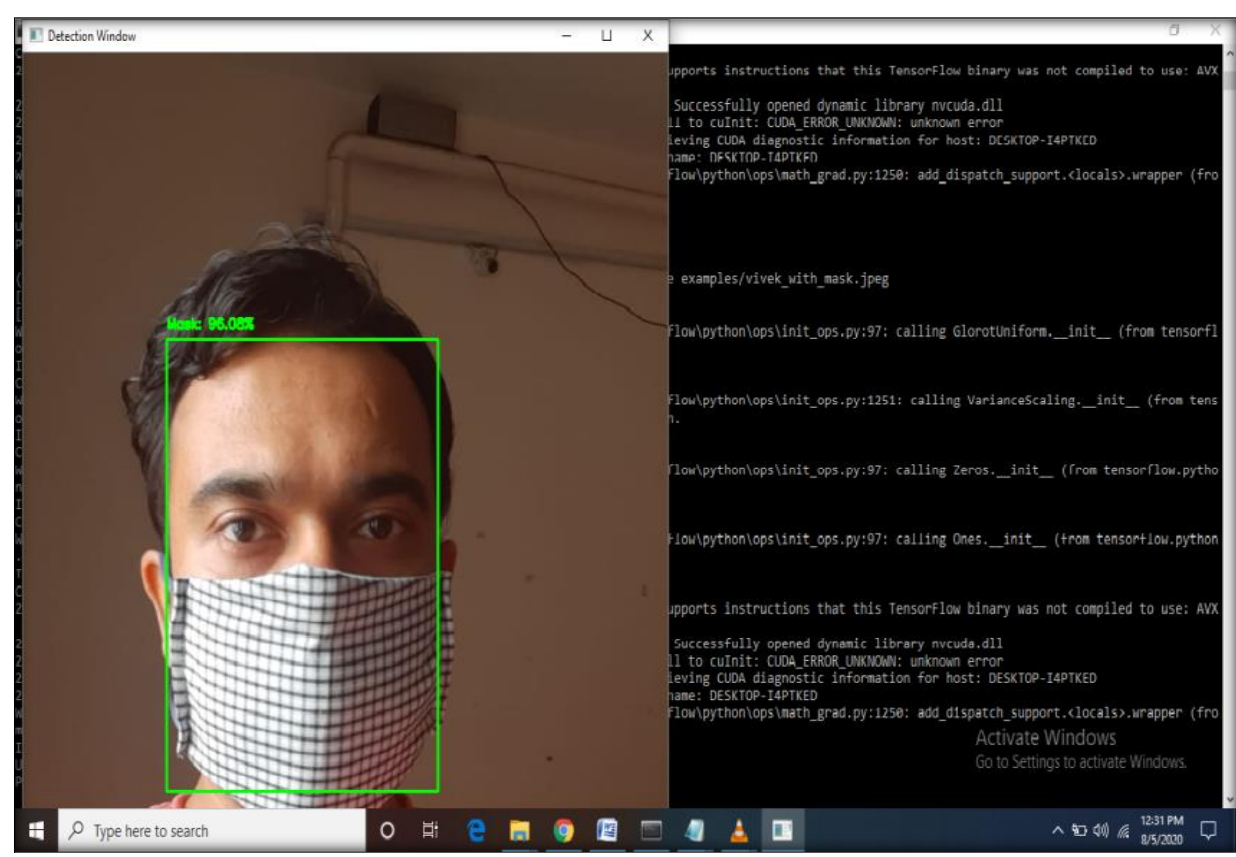

OUTPUT OF THE MODULE

Fig 3

\section{PROBABLE/ PROPOSED APPLICATIONS} are:-

The proposed applications envisaged of the detector

At Entry/ Exit point of establishment. Relieving sentries from additional task.

$>$ Appropriately located points inside offices.

$>$ Offices.

$>$ Mess/ Institutions.

$>$ Walking Plaza

$>$ Shopping Center

$>$ Juice shops

$>$ Auditorium.

$>$ Conference halls.

$>$ Any place where requirement of mask in necessary.

\section{FUTURE ENHANCEMENT/ IMPROVEMENTS}

The model proposed is presently in phase I of operation and the demo video shown is a direct application of it. However in future, following enhancements/ improvements can be superimposed to facilitate the ease of use and simplicity. These are described below:-

Phase-I: Current version uses Laptop with relevant software installed

As such requirement of such loaded laptop with camera is a must for execution of the software. Therefore laptop should be installed at point of inspection itself and some skilled operator should be present to type and execute the relevant commands.
> Phase-II: Replacement of laptop with a compact model with camera attached for video capture

The plan is to replace laptop with a workable model/ device such as Raspberry Pi which needs to be installed at place of inspection/ site. Raspberry Pi models are compact in size. This will reduce installation complexities by $90 \%$. This device/ model will be attached to a laptop only for execution of commands with help of LAN cable. As such commands can be run by a remote operator also and the requirement of laptop present at the site is negated.

Phase-III: Removal of laptop for all purpose. Execution only with help of model

There will not be any constraint of laptop for executing any command to run the model either at the site or at any remote location. The device which is comparable to the size of a credit card with attached camera and loaded with necessary algorithms/code would meet the requirements. There would not be any obligation of trained/ semi trained individuals to run the commands. A normal sentry/ operator should be able to operate the model

\section{CONCLUSION}

Present COVID scenario and rise in COVID cases is itself an alarming situation to deal with. No Vaccine is still available in market. Human trials are still on. Until and unless a viable vaccine is developed and tested, precaution is the only option which can be exercised. As such use of mask is the utmost precautionary measure one can adopt. Lackadaisical attitude of people in wearing masks all the time when one is out of home is something which needs to be monitored. Non usage of MASK by one(positive case of corona but still not detected/asymptomatic) can spread the virus exponentially if the infected is not wearing a mask. Sentry/ Men cannot be deployed everywhere in the establishment to ensure that anyone roaming out is wearing 
mask at all times. All establishment/offices must employ measures to keep a check on the mandate of wearing mask always and every time when out of house. Instead of manual checking, use of technology can be resorted to which not only automates the task of detection but also prevents diversion of substantial manpower to keep a manual check on defaulters. With phase 3 of the future enhancement as described above, a miniature module would be able to perform the mask detection task without need of any skilled operator. This module will not only automate the entire process single handedly but also would be small, compact and easy to install.

\section{REFERENCES}

[1]. Computer vision and deep learning resource guide by Dr. Adrian Rosebrock

[2]. Computer Vision: Algorithms and Applications Richard Szeliski

[3]. Deep Learning by Ian Goodfellow, Yoshua Bengio, Aaron Courville 